\title{
Evolution of the Habitable Zone and Search for Life Around Red Giant Stars, Part I: Interest of the Study.
}

\author{
B. Lopez W. C. Danchi ${ }^{2}$, J.-L. Menut ${ }^{1}$, J. Rajagopal ${ }^{2,3}$, \\ J. Schneider ${ }^{4}$ A. Belu ${ }^{5}$, and R. Barry ${ }^{2,6}$ \\ ${ }^{1}$ Observatoire de la Cote d'Azur, BP 4229, 06304 Nice Cedex 4, France \\ ${ }^{2}$ NASA Goddard Space Flight Center, Exoplanets and Stellar Astrophysics Lab., Code 667, \\ Greenbelt, MD 20771, USA \\ ${ }^{3}$ University of Maryland, Astronomy Department, College Park, MD 20742, USA \\ ${ }^{4}$ Observatoire de Paris, Paris, France \\ ${ }^{5}$ University of Nice - Sophia Antipolis, LUAN, Parc Valrose, 06108 Nice Cedex 2, France \\ ${ }^{6}$ The Johns Hopkins University, Physics and Astronomy Dept., Baltimore, MD 21218, USA
}

\begin{abstract}
We discuss the evolution of the habitable zone around low mass and intermediate mass stars as they evolve off the main sequence. This work shows that this class of stars should be included in the search for life because if planets could be found in their habitable zones, they will allow to test different hypothesis concerning the conditions of life formation. For instance if these planets show traces for life, it is possible to empirically determine a lower limit to the timescale for the formation of life. This time scale is not well determined from the Earth's study, as life formation initially occurred during a period of heavy bombardment from comets and asteroids during the formation of the solar system. We will summarize (to the best of our knowledge) some of the recent results concerning the early signs of life on Earth. We will present some of our work in progress in which we evaluate the effects of stellar evolution on the habitable zone changes.
\end{abstract}

Keywords. instrumentation: planets and satellites: general, high angular resolution, techniques: interferometric, space vehicles: instruments.

\section{Introduction}

Low and intermediate mass stars with masses ranging from 0.5 to $\sim 8 \mathrm{M}_{\odot}$ undergo changes in temperature and luminosity during their evolution. For example, the luminosity of the Sun, which is located on the Main Sequence of the Hertzprung-Russell diagram, currently is 30 percent greater than its zero-age (initial) luminosity (Gough 1981). Other major changes take place after the Sun evolves off the Main Sequence. Along the Red Giant Branch (RGB) the radius of a $1 \mathrm{M}_{\odot}$ star progressively increases up to $\sim 10 \mathrm{R}_{\odot}$, and the luminosity increases up to about $10^{1.5} \mathrm{~L}_{\odot}$ (Maeder \& Meynet 1988). After leaving the RGB a star evolves onto the Asymptotic Giant Branch (AGB). At this stage in its evolution, its radius increases up to a few hundred times $\mathrm{R}_{\odot}$ and the luminosity up to $10^{4} \mathrm{~L}_{\odot}$. The lifetime of a $1 \mathrm{M}_{\odot}$ star, from the moment it leaves the main sequence until the end of the RGB, is of about $2.5 \times 10^{9} \mathrm{yr}$, which can be compared to $\sim 10 \times 10^{9}$ yr spent on the main sequence. The evolution of a star is accompanied by mass loss processes. During the post-main sequence evolution of a $1 \mathrm{M}_{\odot}$ star nearly $0.4 \mathrm{M}_{\odot}$ will be lost to the interstellar medium before it ends its life as a white dwarf (Weidemann 1987). 
The habitable zone gradually moves outward after a star leaves the main sequence. Depending on the length of time that is necessary for life to form, we show that it is possible that life may exist or develop on a planet that is located within a range of 2-9 AU from a $1 \mathrm{M}_{\odot}$ star, during the first stages of its post main-sequence evolution and up to $22 \mathrm{AU}$ from the star during the second stage after the core He flash.

Future space missions, such as TPF (Beichman, Woolf, \& Lindensmith 1999) and Darwin (Fridlund \& Gondoin 2003), that focus on searches of signatures of life on extrasolar planets primarily around main-sequence stars, should also consider the environments of sub-giant and red giant stars. Pathfinder missions such as EPIC (Clampin et al. 2001), Eclipse (Trauger et al. 2003), and FKSI (Danchi et al. 2003a, 2003b) may also be able to observe planets around red giant stars.

Based on a list of selected objects, we show there are adequate numbers of such stars in the Solar neigborhood and that preliminary analysis demonstrates that planets around such stars could be detected without any modifications to present architectural concepts for the interferometer version of TPF and Darwin (see our part II paper, Danchi et al. 2006 presented at this conference).

\section{The evolution of the habitable zone}

From a biological point of view and given the limits of our present knowledge, the existence of life is strongly associated with the presence of liquid water (Brack 1993). Several evaluations of the inner and outer limits of the habitable zone have been proposed, as discussed in the review of Forget (1998). A conservative estimate, minimizing the width of the habitable zone (Forget 1998), assumes that around our own Sun the habitable zone currently ranges from 0.95 to $1.67 \mathrm{AU}$. The inner limit of this conservative estimate is set by an atmospheric water loss effect occurring in the stratosphere of a planet (Kasting et al. 1993, Kasting 1998). The outer limit is set by the lowest temperature at which the liquid/solid phase change of water occurs. The estimate of this outer limit assumes the existence of a greenhouse effect involving $\mathrm{CO}_{2}$ and $\mathrm{H}_{2} \mathrm{O}$ gas (Kasting et al. 1993). A less conservatively defined "habitable zone" extends the outer edge of the limit of the habitable zone to as large as 2.4 AU (Mischna et al. 2000).

A convenient way of estimating the inner and outer limit of the habitable zone at different stages of stellar evolution is to assume that the planet behaves as a gray-body with albedo, $A$, and with perfect heat conductivity (the temperature is uniform over the planet's surface). Its radiative equilibrium temperature, $T_{p}$, is then determined by:

$$
T_{p}=\left[(1-A) L_{\star} /\left(16 \pi \sigma d^{2}\right)\right]^{0.25}
$$

where $L_{\star}$ is the luminosity of the star, $\sigma$ is the Stefan-Boltzmann constant, and $d$ is the distance from the center of the star to the planet. Typically a value of approximately 0.2 is chosen for the albedo to be representative of an earth-like planet. For these calculations we determine the temperature at the limits of the habitable zone, and from this we calculate the inner and outer distances for a given star.

For the conservative limit, we assume an effective equilibrium temperature of $269 \mathrm{~K}$ and $203 \mathrm{~K}$, which for the Sun gives inner and outer limits of 0.95 and 1.37 AU, respectively. For the less conservative limit, the equilibrium temperature is $169 \mathrm{~K}$, so that the outer limit is extended to 2.4 AU. The three equilibrium temperature extrema, defined as the inner and the 2 possible outer limits, can be used to derive the approximate location and width of the habitable zone around a star at different stages of its evolution.

Models describing the stellar evolution are proposed by Maeder and Meynet (1988). For intermediate mass stars having masses from 2 to $5 \mathrm{M}_{\odot}$ the evolution is followed up 
Table 1. Lifetimes (Gyr) of stages of stellar evolution as a function of initial stellar mass

\begin{tabular}{ccccc}
\hline Mass $\left(\mathrm{M}_{\odot}\right)$ & Main Sequence & Subgiant & First Red Giant & Core He Burning \\
\hline 1.0 & 7.41 & 2.63 & 1.45 & 0.95 \\
1.5 & 1.72 & 0.41 & 0.18 & 0.26 \\
2.0 & 0.67 & 0.11 & 0.04 & 0.10 \\
\hline
\end{tabular}

to the end of the early asymptotic giant branch. For stars with masses less than $1.7 \mathrm{M}_{\odot}$, the evolution is calculated up the He-flash occuring at the end of the RGB part of the HR diagram (Iben 1967).

Figure 1(a) displays the time evolution of the inner and outer limits defining the habitable zone assuming the initial mass of the star is $1 \mathrm{M}_{\odot}$. The solid curve represents the evolution of the inner limit of the habitable zone, while the dashed curve depicts the conservative outer limit, and the dotted curve represents the less conservative outer limit.

During the course of stellar evolution, the habitable zone is a shell sweeping progressively outward over a wide range of distances from the star as can be seen in Figure 1(c). The dashed curve represents the duration of the habitable zone for the conservative assumptions for the inner and outer limits of the habitable zone. The dotted curve assumes the same inner limit of the habitable zone but the less conservative outer limit is used. Figure 1(b) displays the evolution of the stellar radius (in units of Solar radii). Immediately after the star leaves the main sequence, the habitable zone progressively moves to 2 AU (Figure 1(c)). The duration of the transit at this location is approximately $10^{9}$ years. A bump/plateau is observed in the curve up to $9 \mathrm{AU}$ (for the conservative limits) and up to $13 \mathrm{AU}$ (for the less conservative limits), where the duration of habitable conditions lasts from a few to several $10^{8}$ years. At $10 \mathrm{AU}$ the duration is smaller, around $10^{8}$ years. At $15 \mathrm{AU}$ from the star, the duration of habitable conditions lasts more than $10^{7}$ years, and at the largest distances the duration gradually decreases. Thus the evolution of the habitable zone at distances up to 25 AU has been considered. During this time period, a $1 \mathrm{M}_{\odot}$ star has not yet reached the AGB.

Although individual masses of red giant stars are not well known in general, and have not been directly determined except in a few cases, Neckel (1975) has shown theoretically that the peak of the red giant mass distribution occurs at progenitor masses of about $1.1 \mathrm{M}_{\odot}$. An empirical estimate of red giant masses determined by Scalo, Dominy, and Pumphrey (1978) is in agreement with the theoretical estimate, with values $0.8-1.2 \mathrm{M}_{\odot}$. This is fortunate since stars selected in a magnitude-limited or distance-limited survey will therefore mostly likely be low mass stars that have long habitable zone lifetimes similar to those calculated in Fig. 1.

Also, it is important to note that the calculations in this paper are focused on the evolution of the habitable zone from the time period after the star evolves off the main sequence up the first ascent along the Red Giant Branch during which the star is burning hydrogen in a shell around a growing He core, i.e., only to the point of the helium flash. After the helium flash, there is an additional long-lived phase of quiescent core helium burning with nearly constant luminosity.

Scalo and Miller (1979) developed interpolation formulae for the lifetime of a star at its various stages of evolution: main sequence, subgiant, first red giant, and first and second helium burning phases, as a function of initial stellar mass. For completeness we display the lifetimes computed from their formulae for stellar masses of $1.0,1.5$, and $2.0 \mathrm{M}_{\odot}$, which we display in Table 1. 

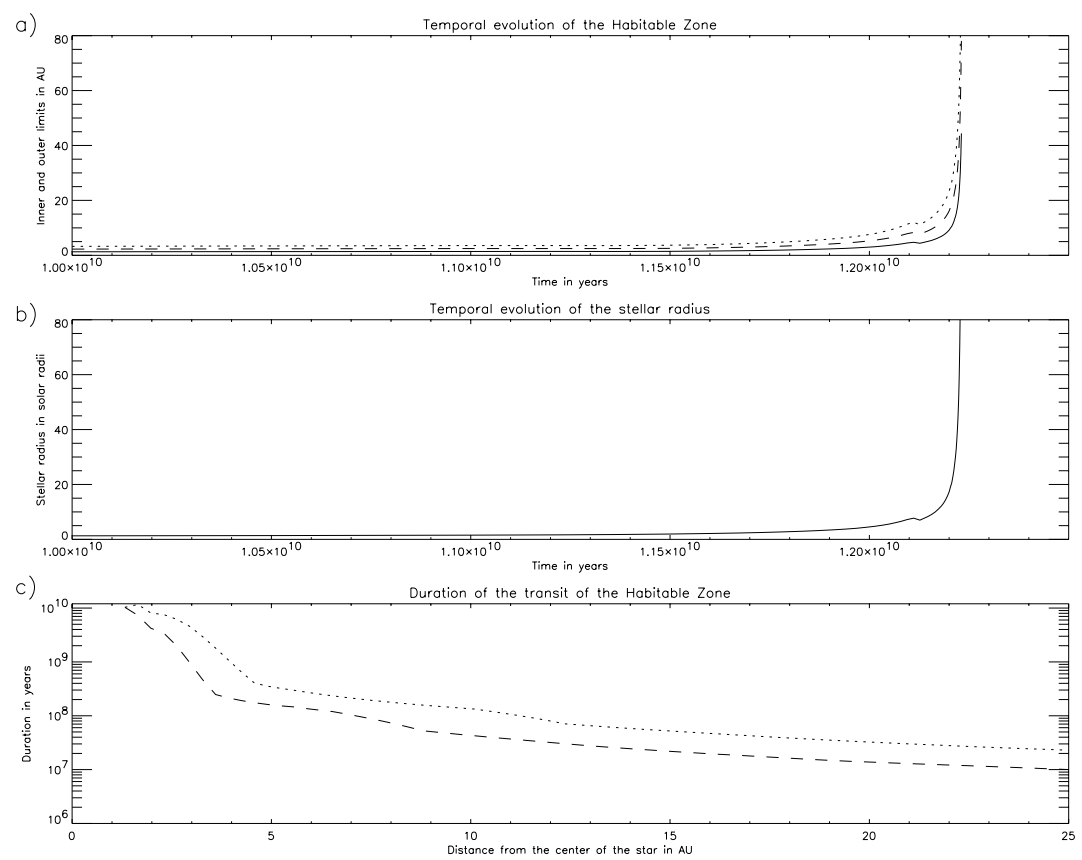

Figure 1. a) The evolution of inner and outer limit of the habitable zone around a post-main sequence star of $1.0 \mathrm{M}_{\odot}$. The time scale displayed is between 12.5 and 12.8 Gyrs. b) The corresponding evolution of the stellar radius (from Maeder and Meynet 1988). c) The duration of the transit of the habitable zone represented at different distances from the star.

For a $1.0 \mathrm{M}_{\odot}$ star, core helium burning provides a nearly constant luminosity phase, adding an additional $10^{9}$ years to the duration of the habitable zone for low mass stars, with substantial increases to the lifetime of the habitable zone for higher mass stars as well. The luminosity during the core helium burning phase is approximately $2.3 \times 10^{2} \mathrm{~L}_{\odot}$, $9.3 \times 10^{2} \mathrm{~L}_{\odot}$, and $2.3 \times 10^{2} \mathrm{~L}_{\odot}$, for $1.0,1.5$, and $2.0 \mathrm{M}_{\odot}$ stars, respectively (Kozlowski \& Paczynski, 1975). Thus an additional period of habitability exists for the region between 7 and $22 \mathrm{AU}$ for a $1 \mathrm{M}_{\odot}$ star during core helium burning.

We summarize our analysis of the evolution of the habitable zone by noting that there are two opportunities for life in the extended habitable zones around evolved stars. Initially life has an opportunity as the star slowly evolves off the main sequence. After the first ascent along the RGB and the He core flash, there is a second chance during the core He burning phase. This latter phase is particularly interesting because of the nearly constant luminosity of the star under these conditions.

\section{The possibility of the existence of life around Red Giant Stars}

The Earth is known to have formed about 4.55 Gyr ago. Substantial evidence exists that life on Earth occurred at least as far as 3.85 Gyr ago rests on the basis of isotopic ratios of carbon residues within grains of apatite (Mojzsis et al. 1997, Holland 1997). 
Rocks older than 3.60 Gyr are all metamorphosed at a high grade and most are strongly deformed, thus morphological fossils if originally present are unlikely to be preserved (Rosing 1999). The oldest purported fossils of microorganisms have an age of $3.50 \mathrm{Gyr}$ and may represent photosynthetic cyanobacteria (Schopf 1993, 1994). The findings and interpretation of this work was recently debated (Brasier et al. 2002, Dalton 2002).

Based on these considerations, it is possible for life to have existed at least as long ago as $3.85 \mathrm{Gyr}$, but this is uncertain because of the imprecise knowledge of the ages of the rocks, and ambiguous interpretations of the isotopic ${ }^{12} \mathrm{C} /{ }^{13} \mathrm{C}$ ratios. Thus, for our discussion, we consider three time scales for life to form, a conservative estimate of $1 \mathrm{Gyr}$, and a less conservative one of $0.5 \mathrm{Gyr}$, and an optimistic one of 0.1 Gyr. Recent analyses of isotopic ${ }^{13} \mathrm{C}$ ratios on micron-scale mineralized tubes in Archean pillow lavas by Furnes et al. (2004) suggest that microbial life colonized these oceanic volcanic rocks soon after they were formed approximately 3.5 Gyr ago. This provides some further support for the optimistic time scales of a few hundred million years or less for life formation.

Dating of terrestrial fossils reveals that life had evolved on Earth by the end of the period of heavy bombardment. Evidence for the bombardment comes from theoretical studies of planetary formation and directly from lunar cratering. Heavy bombardment may have helped set the stage for the terrestrial origin of life by delivering key biogenic elements (H, C, N) to the Earth's surface (Chyba et al. 1994). For its first 800 Myr, the Earth's surface would have been subjected to frequent impacts, possibly delaying the development and spread of life (Maher and Stevenson 1988).

Due to the rapid evolution of a star once it has left the main sequence, the transit time of the habitable zone shortens. For an evolved $1 \mathrm{M}_{\odot}$ star, the transit of the habitable zone crossing a planet at $2 \mathrm{AU}$ of distance lasts slightly more than $10^{9}$ years, a time scale clearly larger than the time which has been required for the emergence of life on Earth.

At about $5 \mathrm{AU}$ of distance, the duration of habitable conditions lasts from $10^{8}$ years to several $10^{8}$ years. It is about one order of magnitude shorter than the time estimated for occurrence of life on Earth, however this time scale may be long enough for life to develop. It represents an interesting test case for future observations.

Effects of the stellar mass loss phenomena and tidal dissipation between the planets and the red giant star are not negligible in the dynamics of the orbits (Rasio et al. 1996). When a Sun like star reaches a radius of about $100 \mathrm{R}_{\odot}$, only a little more than $10 \%$ of the initial mass of the star has been lost, and the effect of this mass loss among the dynamical changes of the planet orbits (for planets more distant than 1 AU not yet subject to tidal effects) is to increase by $10 \%$ the semi-major axis. It is interesting to note that the increase of the semi-major axis, if occuring for a planet during a time when it is in the habitable zone (itself expanding), will tend to increase the time duration of habitable conditions for the planet.

\section{Conclusions}

We have estimated the time transit of the habitable zone around evolved stars of initial mass $1.0,1.5$ and $2.0 \mathrm{M}_{\odot}$ from the time period after which a star moves off the main sequence until it reaches the point of the He flash (see for more details Lopez et al. 2005). The temporal transit of the habitable zone is estimated to be of the order of several $10^{9}$ years for a planet at $2 \mathrm{AU}$ and around $10^{8}$ years for a planet at $9 \mathrm{AU}$. Furthermore, after the star ascends the Red Giant Branch for the first time and undergoes the He flash, there is an additional long stable period of core He burning $\sim 10^{9}$ years during which the habitable zone ranges from 7 to $22 \mathrm{AU}$ from the star. 
We have shown that the evolution of the habitable zone as stars move off the main sequence and onto the subgiant and later the red giant branches of the HR diagram provide a new opportunity for the search for Earth-like planets and life. For these stars life has an opportunity to evolve under conditions with much less bombardment than existed in the early Solar system. We also show (Danchi et al. 2006 this proceeding) there are adequate numbers of such stars in the Solar neigborhood, and that preliminary analysis demonstrates that planets around such stars can detected without any modifications to present architectural concepts for the interferometer version of TPF and Darwin.

\section{Acknowledgements}

We acknowledge support from : CNRS; GSFC Internal Research and Development and Director's Discretionary Funds; a Michelson Fellowship and a National Research Council (NRC) Fellowship.

\section{References}

Beichman, C., Woolf, N.J. \& Lindensmith, C., ed. 1999 (JPL Publ. 99-3; Pasadena: Caltech)

Brack A. 1993, 'Origine of Life', 3, 10

Brasier, M.D. et al. 2002, LPI, 33, 1614

Chyba C.F., Owen T.C. \& Ip W.-H., 1994, 'Hazards due to Comets and Asteroids', T. Gehrels Editor, The University of Arizona Press

Clampin, M., Ford, H.C., Illingworth, G. \& Petro, L. 2001, BAAS, 33, 1365

Dalton, J.B. 2002, LPI, 33, 1555

Danchi W.C., Deming D., Kuchner M.J. \& Seager S. 2003a, ApJ, 597, L57

Danchi, W.C., et al. 2003b, in the Proceedings "Towards Other Earths: Darwin/TPF and the Search for Extrasolar Terrestrial Planets," Heidelberg, Germany, 22-25 April 2003, ESA Publication SP-539, p. 83

Forget, F. 1998, Earth, Moon, Planets 81, 59

Fridlund, C.V.M. \& Gondoin, P. 2003, Proc. SPIE, 278, 1273

Furnes, H., Banarjee, N.R., Muelenbachs, K., Staudigel, H. \& de Wit M. 2004, Science, 304, 578

Gough D.O. 1981, Solar Physics 74, 21

Holland H.D. 1997, Science 275, 38

Iben I. 1967, ARAA 5, 571

Kasting, J., Whitmire, D.P., \& Reynolds, R.T. 1993, Icarus, 101, 108

Kasting J.F. 1998, AAS 193, 5003

Lopez, B., Schneider, J., \& Danchi, W.C. 2005, ApJ, 627, 974

Maeder, A. \& Meynet, G. 1988, A\& AS, 76, 411

Maher K.A. \& Stevenson, D.J., 1988, Nature 331, 612.

Mischna, M.A., et al. 2000 Icarus, 145, 546

Neckel, H. 1975, A\&A, 42, 379.

Rasio, F.A., Tout, C.A., Lubow, S.H. \& Livio, M. 1996, ApJ 470, 118

Rosing M.T., 1999, Science 283, 674

Scalo, J.M., Dominy, J.F., \& Pumphrey, W.A. 1978, ApJ, 221, 616

Scalo, J.M. \& Miller, J.E. 1979, ApJ, 233, 569

Schopf, J.W. 1993, Science, 260, 640

Schopf, J.W. 1994, Proc. Natl. Acad. Sci. USA 91, 6735

Schröder, K.-P. \& Sedlmayr, E., 2001, 366, 913

Trauger, J., et al. 2003, Proc. SPIE, 4854, 116

Weidemann, J. 1987, A\&A, 188, 74 